\title{
La privatización de la educación en Chile: análisis de los discursos del profesorado y de la dirección de los centros sobre la calidad de la educación y la rendición de cuentas
}

The privatization of education in Chile: a comparative analysis of teachers' and principals' discourses about education quality and accountability Ignacio Eduardo Reyes Cayul, Adbeljalil Akkari ${ }^{1}$

\section{Resumen}

En materia de privatización de la educación, Chile suscita el interés y controversias a nivel Latino-americano. En efecto, el proceso de privatización se inició y tuvo una aceleración bajo el régimen militar, especialmente desde inicio de la década de los ochenta; manteniéndose prácticamente durante la transición democrática, lo que incluye ciertamente a los gobiernos de centro-izquierda. En Chile, asistimos, como en otros países latino-americanos, a una verdadera indefinición de fronteras entre lo público y lo privado en beneficio de grupos sociales favorecidos. Mediante entrevistas semi-dirigidas, hemos recabado la opinión de directivos y profesores de escuelas particulares subvencionadas y municipales de tres comunas de la Región Metropolitana de Santiago. Expondremos que el discurso de los actores de la educación (docentes y directores) se distancia de la calidad y de la rendición de cuentas definidas por la política educativa, cuando las escuelas se ven confrontadas a la pobreza extrema y a la violencia. En estos casos, el simple hecho tener a los niños en la escuela y relacionarse en un ambiente de paz, son desde ya, resultados en sí mismos, más allá de toda tentativa burocrática de medición de resultados de aprendizaje. Al mismo tiempo, la profesión docente muestra una faceta distinta, toda vez que se enmarca en la dinámica interna de las escuelas, atendiendo a las necesidades que el contexto de pobreza demanda.

\section{Palabras claves}

Privatización, público-privado, calidad, rendición de cuentas, gestión, profesión docente

\section{Abstract}

In terms of the privatization of education, Chile raises interest and controversies in Latin America. The privatization process started at the beginning of the 1980's under the military regime and spread very quickly. It kept on going during the democratic transition, as well as at the times of the center-left governments. In Chile, as in other Latin American countries, the difference between public and private education is not well defined and that is to the benefit of the upper classes. Through semi-structured interviews, we have obtained the opinion of managers and teachers of subsidized and municipal private schools in three communes of the Metropolitan Region of Santiago. We will explain the difference between the notions and the standards of quality and accountability of the educational actors (teachers and principals) and those defined by the educational policy, in particular in those cases where schools are confronted with extreme poverty and violence. In such cases, simply having children at school and interacting in a peaceful environment are results in themselves, beyond any bureaucratic attempt to measure learning outcomes. At the same time, the teaching profession shows a different face, since it is part of the internal dynamics of schools, attending to the needs that are specific to a context of poverty.

Key words

Privatization, public-private, quality, accountability, management, teaching profession. 


\section{Introducción}

En el presente artículo nos interesaremos por una de las facetas de la privatización de la educación en Chile constituida por la proliferación y auge de las escuelas particulares subvencionadas ${ }^{2}$ desde la década de los ochenta en adelante. En el primer apartado, observaremos cuáles han sido las políticas educativas impulsadas en estas cuatro décadas que nos permiten hablar de un auge de las escuelas privadas subvencionadas; del mismo modo, nos adentraremos en los cambios experimentados por el Estado, a lo largo del periodo de tiempo mencionado. Para este efecto nos apoyaremos en destacadas investigaciones nacionales e internacionales. En el segundo y tercer apartado, abordaremos, una forma de privatización, llamada en la educación o privatización endógena. Ball y Youdell (2007) mencionan un conjunto de prácticas, principios, creencias importadas desde la esfera privada y aplicadas en las instituciones públicas, en plano educativo chileno, nos referimos a los organismos gubernamentales y las escuelas municipales. Sin embargo, este tipo de privatización endógena, se vincula estrechamente con la noción de hibridación de fronteras público-privado, esto es, tal como lo mencionamos, la aplicación de modelos del mundo privado en las organizaciones públicas; pero también la importación de principios y valores históricamente asociados a la esfera pública pero aplicados ahora, por instituciones privadas. De este modo, asistimos a una diversificación nunca antes vista de las formas de privatización, las cuales funcionan en distintos planos y temporalidades, haciendo cada vez más difícil establecer una clara diferencia entre instituciones públicas y privadas. En el tercer apartado presentaremos los resultados de un trabajo de campo, efectuado en 2016, sobre escuelas municipales y particulares subvencionadas de la Región Metropolitana de Santiago ${ }^{3}$; nos centraremos en el discurso de los docentes de estas escuelas sobre la calidad de la educación y la rendición de cuentas, poniendo en evidencia las convergencias y divergencias entre profesores. En la cuarta parte, analizaremos el modo de gestión dominante en los dos tipos de establecimiento, a través del discurso de directores de escuela; a su vez, intentaremos retratar la reconfiguración de la profesión docente en Chile impulsada por mecanismos de competición entre escuelas públicas y privadas. Finalmente, ofrecemos una discusión final sobre las principales ideas surgidas en los distintos apartados del presente artículo.

\section{Auge de las escuelas particulares subvencionadas dentro de un proceso de privati- zación del sistema escolar chileno.}

El sistema educativo chileno vivió un cambio significativo durante la dictadura militar. A inicios de los ochenta, se plasman reformas tanto a la organización de la estructura escolar como en los planes y programas de estudios. Ante la creciente demanda de escolarización de la población, el régimen optó por una masificación de las escuelas mediante la apertura a proveedores privados del servicio educativo, llamados sostenedores o cooperadoras del rol educador del estado (Almonacid, 2008: 164). Así, el estado pasó a subvencionar la matrícula de estas escuelas mediante vouchers o unidades de subvención escolar. En este tiempo, el crecimiento de las escuelas particulares subvencionadas fue de $15 \%$ a un $30 \%$. Este periodo llamado de Impulso inicial (Larrañaga, 2004: 4), la dictadura sentó las bases para la entrada de privados. Al hablar de impulso inicial, es preciso mencionar qué se entiende por esto y fundamentalmente, quién lo posibilitó. Falabella (2015: 703-709) llama a este periodo Estado subsidiario y antiestatismo, pues de lo que se trata es de comprender cómo el Estado abandona su rol de proveedor y administrador de las escuelas, para pasar a

2 Escuelas particulares subvencionadas: denominación usada en Chile para referirse a las escuelas privadas.

3 Este trabajo de campo forma parte de la investigación: "Privatización de la educación en Chile: estudio de la caso de la escuela privada en sectores vulnerables". Tesis de doctorado en ciencias de la educación de la Universidad de Ginebra, realizada por Ignacio Reyes Cayul, bajo la dirección del profesor Abdeljalil Akkari, miembros del equipo de investigación ERDIE, Équipe de Recherche en Dimensions Internationales de l'Education. 
La privatización de la educación en Chile: análisis de los discursos del profesorado y de la dirección de los centros sobre la calidad de la educación y la rendición de cuentas

delimitar el marco legal escolar basado en un cuasi-mercado escolar donde el Estado financia la demanda educativa (elección de las familias). Recordemos que en 1980, se dicta la nueva Constitución de la República, en la cual se consagra el derecho de abrir, organizar y mantener establecimientos educacionales. Al mismo tiempo, mediante el Decreto Ley 3477 del mismo año se traspasan las escuelas fiscales a las municipalidades. Concurriendo al mismo sistema de subvención a la demanda. Con estas dos medidas se produjo el encuentro entre la delegación, último eslabón de la descentralización, y la privatización o apertura a privados en el campo educativo. El voucher a escuelas particulares y municipalidades constituyen formalmente el sector subvencionado de educación (recordemos que existe también las escuelas particulares pagadas, financiadas integralmente por las colegiaturas de las familias que atiende al $9 \%$ de la matrícula escolar) ${ }^{4}$. Por lo tanto, este nuevo modelo de cuasi-mercado escolar quedó constituido, por un lado, con sostenedores privados (en 2004, existían 4229 escuelas; en 2013, la cifra llega a 6017), produciéndose una expansión de este sector del orden del $26 \%$ de la matrícula nacional. La municipalización, por otro lado, rediseñó los territorios escolares, multiplicando el número de administradores o sostenedores públicos (345 municipalidades), generando en cada uno de estos municipios cuasi-mercados escolares ${ }^{5}$ : las escuelas particulares subvencionadas y municipales compiten por la matrícula. En este nuevo escenario el Estado financia y evalúa (en este tiempo fundamentalmente la entrada de un nuevo operador al sistema), tomando el rol subsidiario ya señalado.

A finales de la dictadura militar e inicios de la llamada transición a la democracia, marcada por los dos primeros gobiernos de la Concertación $(1990$ - 2000), se introducen intervenciones al sistema escolar, atendiendo a medidas de equidad. Los llamados programas compensatorios de apoyo técnico y asistencia a escuelas (Cox, 2003), se enfocaron en los casos con más bajo rendimiento (el programa de la novecientas escuelas o P900) ${ }^{6}$; la extensión de textos escolares, construcción de bibliotecas e infraestructura (Los programas de mejoramiento de la calidad y equidad de la educación o MECE-Básica y MECE-Media) ${ }^{7}$; , la introducción de las nuevas tecnologías de la información, implementado laboratorios de computación en las escuelas de menores recursos (programa o Red ENLACES) ${ }^{8}$. El objetivo de estas políticas fue focalizar los recursos y la acción del Ministerio de Educación en los establecimientos escolares que contasen con bajos recursos y se centraron en las ideas de equidad escolar, es decir, apoyar, desde el Estado, a los establecimientos que atienden en los sectores más pobres del país; y de efectividad escolar, esto es, la obtención de mejores resultados escolares pese a contar con condiciones de funcionamientos precarias. La articulación de equidad y efectividad fueron el eje de este conjunto de políticas compensatorias.

Para fines del siglo veinte, la calidad de la enseñanza ocupa el discurso público, así como las acciones del Ministerio de Educación. Es preciso señalar, sin embargo, que en 1995 se producen dos medidas importantes: por un lado, se publican por primera vez en la prensa nacional, los resultados de la prueba

\footnotetext{
4 Fuente: MINEDUC 2013.

5 La literatura sobre cuasi-mercados escolares identifica varios tipos de espacios donde las escuelas compiten por la matrícula: según la ubicación en un mismo territorio, municipal o incluso regional; entre escuelas privadas; entre escuelas públicas; según el tipo de proyecto educativo. Para más información sobre este tema, consultar los trabajos de Torres (2000), Almonacid (2004; 2008), Córdoba, (2014).

6 Programa de Mejoramiento de la Calidad de las Escuelas Básicas de Sectores Pobres (P900): Desde 1990 a la fecha, este programa se propone apoyar al $10 \%$ de las escuelas con peor rendimiento y mayores necesidades, para que los alumnos logren dominar las destrezas culturales básicas: lectura, escritura y matemática elemental (Fuente: http://www.cepal.org/cgi-bin/getProd.asp?xml=/prensa/noticias/comunicados/5/6385/P6385.xml\&xsl=/prensa/tpl/p6f.xsl\&base=/prensa/ tpl/top-bottom.xsl)

7 MECE-Básica/MECE-Media: "Programa de Mejoramiento de la Calidad y Equidad de la Educación Básica". Amplió sustancialmente la entrega de textos en este nivel, se inició la distribución de las bibliotecas de aula y se incluyeron fondos para aumentar la cobertura en educación parvularia.

8 Red ENLACES: Considera la instalación de tecnología informática de punta en todos los establecimientos.
} 
La privatización de la educación en Chile: análisis de los discursos del profesorado y de la dirección de los centros sobre la calidad de la educación y la rendición de cuentas

SIMCE'; por otro lado, se introduce el Financiamiento Compartido, el pago de colegiaturas por parte de las familias en las escuelas subvencionadas (Particulares y municipales, aunque en estas últimas, se debe hacer una consulta a los apoderados). En el caso del SIMCE, este indicador comienza a cobrar fuerza para los gobiernos, posicionándose como criterio (excluyente) de calidad. Las movilizaciones estudiantiles de 2006 y 2011, instalan definitivamente la calidad de la educación como el eje más importante del sistema educativo chileno. Los gobiernos de Piñera (2010 - 2014) y Bachelet (2006 - 2010; 2014 - 2018) han enfocado esta petición estudiantil desde una fuerte estandarización del sistema escolar, abarcando muchos ámbitos: medición de los aprendizajes, gobernanza de las escuelas, liderazgo directivo, entre otras. La publicación del marco para la buena enseñanza y para directores ${ }^{10}$; la política de evaluación docente $^{11}$. El Estado pasa a regular la calidad de la educación incrementando su aparato de supervisión (Se crea la Superintendencia de Educación y la Agencia de la Calidad) ${ }^{12}$.

$\mathrm{El}$ auge de las escuelas particulares subvencionadas ha pasado por un reacomodo del rol y naturaleza del Estado, pasando de ser un ente subsidiario, a uno compensatorio y finalmente, vigilante de la calidad. No obstante, no hay que entender este proceso como una sustitución de roles sino más bien como la interacción entre todos. Siendo el Estado supervigilante (Falabella, 2015: 703-709) una reorganización de la subsidiariedad y medidas compensatorias, por lo tanto, la matriz del sistema escolar, el cuasi-mercado escolar no ha experimentado un cambio a su estructura, sino que el Estado ha garantizado su existencia, al tiempo que ha establecido los resultados en test estandarizados como factor que determina la calidad.

\section{Privatización endógena o en la educación e hibridación de fronteras público - pri- vada.}

El proceso de privatización chileno pone de manifiesto las formas clásicas de este fenómeno, es decir, determinar su grado según la propiedad de las instituciones y del financiamiento: el postulado dice que las escuelas se definen por la propiedad y la fuente del financiamiento. Pues bien, el caso chileno muestra que los mecanismos de financiamiento, privados y públicos, las prácticas como la rendición de cuentas y la autonomía de la gestión, han sido ejecutadas siguiendo lógicas venidas desde el mundo privado (Luengo y Saura, 2013), al tiempo que son comunes a ambos tipos de escuelas. Es importante, a este nivel, renovar nuestros enfoques de análisis sobre la privatización poniendo en un primer lugar la noción de privatización endógena (Ball \& Youdell, 2007); para continuar con la idea de hibridación de fronteras entre lo público y lo privado (Akkari et al., 2011).

\footnotetext{
9 SIMCE: Sistema de Medición de la Calidad de la Educación, test que mide los conocimientos en lectura, escritura y matemática (Además de Historia, Ciencias Naturales, actualmente, inglés y educación física). Esta prueba, de carácter nacional, se efectúa en $2^{\circ}, 4^{\circ}, 6^{\circ}$ y $8^{\circ}$ año de educación básica; y en II y IV año de enseñanza media.

10 Marco para la buena enseñanza: Referente sobre el desempeño profesional de docentes de los sistemas escolares. (Fuente: https://www.google.ch/url?sa $=\mathrm{t} \& \mathrm{rct}=\mathrm{j} \& \mathrm{q}=\& \mathrm{esrc}=\mathrm{s} \&$ source $=$ web\&cd $=1 \& \mathrm{ved}=0 \mathrm{ahUKEwjpnLurgcrUAhUPfFAKHWsNCogQFggnMAA \& url}=\mathrm{https} \% 3 \mathrm{~A} \% 2 \mathrm{~F} \% 2 \mathrm{Fwww} . \mathrm{docentemas}$. cl\%2Fdocs\%2FMBE2008.pdf\&usg=AFQjCNFxTAi3vDPY2yGZzdp91ct9CS224A\&sig2=MryUJEvFEyuPDbfG01Ak8g\&cad=rja). Marco para la buena dirección: referente que define las prácticas, competencias y conocimientos para el desarrollo del liderazgo escolar en el país. (Fuente: http://liderazgoescolar.mineduc. $\mathrm{cl} /$ marco-para-la-buena-direccion-y-el-liderazgo-escolar/)

11 Evaluación docente: El Sistema de Evaluación del Desempeño Profesional Docente está orientado a promover el desarrollo profesional continuo y a mejorar la labor pedagógica de las y los profesionales de la educación que se desempeñan en funciones docentes de aula. (Fuente: http://www.cpeip.cl/evaluacion-docente/).

12 En 2008 se aprueba la ley de General de Educación que crea la Superintendencia de Educación y la Agencia de la Calidad. Esta nueva estructura se refrenda en 2011 con la ley de Aseguramiento de la calidad.
} 
La privatización de la educación en Chile: análisis de los discursos del profesorado y de la dirección de los centros sobre la calidad de la educación y la rendición de cuentas

\subsection{Privatización endógena}

Conocida también como privatización en la educación o formas camufladas de privatización. Este nuevo enfoque supone una gran transformación del ethos de las instituciones públicas.

Por privatización endógena nos referimos a todos los mecanismos, prácticas y valores nacidos en el mundo privado, desde la empresa privada e importados hacia la esfera pública. Esta forma de privatización no afecta, sin embargo, la naturaleza de las instituciones - por tanto, no hablamos de traspasos directos de propiedades públicas a privadas, en este caso, debemos hablar de formas de privatización exógena o de la educación ${ }^{13}$ - por tanto, la escuela no pierde su estatus público (no obstante, lo público no es administrado directamente por el Estado central sino que, en el caso chileno, la escuela pública fue descentralizada hacia las municipalidades). Cuando nos referimos a mecanismos importados desde el mundo privado hablamos de objetivos, evaluaciones, contratos, proyectos, dispositivos que se encuentran en diversas áreas del quehacer escolar ${ }^{14}$. De este modo, se generó una política pública basada en resultados medibles en función de rendimientos sostenidos en el tiempo, dando paso a una escuela llamada efectiva (Bellei et al., 2003; 2014) y a un paradigma de la calidad basado en la performatividad (Ball, 2003; Luengo y Saura, 2013), de manera que, tanto la modelización de las políticas educativas como los dispositivos internos de las escuelas (planificación de los aprendizajes de los alumnos basados en resultados en pruebas estandarizadas) se dan en este enfoque.

\subsection{Hibridación de fronteras público-privado}

Las formas endógenas de privatización suponen la adopción de dinámicas del mundo privado, en el plano público. Sin embargo, la literatura ha mostrado transferencias de mecanismos, prácticas y valores de un sector a otro. La hibridación de fronteras (Akkari et al., 2011) hace referencia a la ida y vuelta conceptual y de las prácticas concebidas en un sector pero adoptadas, con el paso del tiempo en otros contextos, siempre en el plano público y privado. Sin embargo, cuando hablamos de hibridaciones, no hablamos de una adopción mecánica de procedimientos, sino de una reinterpretación de éstos, según cada institución. Se confirma la definición inicial de Ball y Youdell (2008) sobre la característica dinámica de la privatización. Así, dentro de un cuasi-mercado escolar, existen establecimientos que apuestan por obtener mejores resultados escolares en un contexto nacional; y otros que se hacen cargo de otros desafíos, como por ejemplo, bajar las tasas de abandono escolar o aumentar sus resultados según su propio historial. En ambos casos, la racionalidad que opera es la efectividad de estos procesos, buscando la adecuación entre los recursos presupuestarios disponibles y la armonización de sus proyectos educativos, conformación de los planteles escolares y plantas docentes.

Por último, estos dos ejemplos se encuentran tanto en escuelas particulares públicas como privadas: el caso de establecimientos municipales que buscan altos scores en test estandarizados, entendiendo este objetivo como la performatividad (Ball, 2003; Luengo y Saura, 2013), del proceso educativo, por ende, venida desde un pensamiento neoliberal de la educación (Carnoy, 1998; Torres, 2000); mencionamos el caso de escuelas particulares subvencionadas que asumen la disminución del abandono escolar como proyecto propio entendiendo este objetivo como una urgencia pública.

\footnotetext{
13 Privatización exógena o de la educación se refiere a la trasferencia total o parcial de la propiedad de una escuela, así como el cobro de matrículas y colegiaturas a las familias, entre otras formas. Para mayor detalle, ver el trabajo de Ball y Youdell (2008).

14 Un ejemplo emblemático de la política educativa es la Ley de Subvención escolar preferencia de 2008. Esta ley introdujo un aumento de la subvención escolar por concepto de matrícula de alumnos prioritarios o vulnerables: de este modo, la asignación de estos fondos se enmarcan en la equidad (aumentar la cuota de este tipo de alumno), y fundamentalmente, en la efectividad, es decir, mejorar los resultados SIMCE. El Ministerio de Educación establece una clasificación de escuelas según los resultados obtenidos: autónomos, emergentes, en recuperación. (Fuente: https://www.ayudamineduc.cl/ficha/clasificacion-de-establecimientos-sep-10).
} 


\section{Calidad y rendición de cuentas según docentes de escuelas municipales y particu- lares subvencionadas.}

En esta cuarta parte, nos centraremos en el discurso de docentes de escuelas municipales y particulares subvencionadas sobre la calidad de la educación y la rendición de cuentas. Estos datos fueron recogidos durante una investigación de campo sobre escuelas en sectores de pobreza en 2016.

Nuestra investigación se realizó en la Región Metropolitana de Santiago. Las comunas seleccionadas fueron Quilicura, Recoleta y La Pintana. De ellas, dos escuelas particulares subvencionadas se encuentran en Quilicura; una particular subvencionada y una municipal en Recoleta; además de una particular subvencionada y una municipal en La Pintana.

Hemos realizado dieciocho entrevistas semi-estructuradas: seis a directores, tres mujeres y tres varones; doce a docentes, de los cuales diez son profesoras mujeres y dos son profesores hombres; todos de nivel básico de lengua castellana y matemática. Las entrevistas fueron íntegramente transcritas. La elección de estas escuelas se hizo en base a su ubicación próxima a poblaciones ${ }^{15}$ : Las poblaciones San Luis y Parinacota de Quilicura; La Pincoya y la Chimba en Recoleta y la población El Castillo en La Pintana. Hemos seleccionado algunos párrafos del capítulo octavo de nuestra investigación antes nombrada y que se enmarca en el trabajo de tesis doctoral de uno de los autores del presente artículo.

Estos tres contextos reflejan una presencia de la escuela particular subvencionada más elevados que los niveles nacionales: el porcentaje nacional de alumnos escolarizados en este sector llega al 54,6\%. En las tres comunas seleccionadas, la realidad es más pronunciada en favor de las escuelas particulares subvencionadas: en Quilicura, el alumnado en estas escuelas llega al 79,5\%; en Recoleta al 62,9\%; finalmente en La Pintana llega al 82,2\% (MINEDUC, 2015).

Con el fin de mantener la confidencialidad de las escuelas y actores visitados, hemos asignado una codificación a cada escuela que detallamos a continuación:

Tabla I. Codificación de las entrevistas

\begin{tabular}{ccc}
\hline CODIFICACIÓN ESCUELA & TIPO & COMUNA \\
\hline Part-Subvl & Particular subvencionada & Quilicura \\
\hline Part-Subv2 & Particular subvencionada & Quilicura \\
\hline Part-Subv3 & Particular subvencionada & Recoleta \\
\hline Part-Subv4 & Particular subvencionada & La Pintana \\
\hline Munl & Municipal & Recoleta \\
\hline Mun2 & Municipal & La Pintana \\
\hline
\end{tabular}

Fuente: elaboración propia.

15 Poblaciones: nombre de los barrios pobres en Chile, ubicados en la periferia urbana. Este apelativo es la continuación de las llamadas poblaciones callampas: ocupaciones de terrenos fiscales o privados, realizadas por obreros, peones, gañanes y familias de sectores populares, migrantes del campo a la ciudad, que, ante la escasez de viviendas sociales y falta de recursos para el arriendo, se asentaron en estos terrenos.

- San Luis y Parinacota: comuna Quilicura, ubicada en sector norte de la Región Metropolitana;

- La Pincoya y la Chimba: comuna de Huechuraba y Recoleta respectivamente ubicada en sector Centro - norte de la Región Metropolitana;

- El Castillo: comuna de La Pintana, ubicada en sector sur de la Región Metropolitana. 
En este apartado sobre la calidad, nos concentraremos en el discurso del profesorado.

\section{1. La calidad de la educación}

Para entender con mayor detalle este punto, nos adentraremos en tres ejes que reúnen la mayoría de las consideraciones de los docentes, a saber, aspiraciones a corto plazo; convivencia escolar; y SIMCE.

\subsection{Calidad de la educación: aspiraciones en el corto plazo}

En una escuela particular subvencionada, los lazos que se crean con los niños se perciben como los más importantes. Este factor comprende elementos que van más allá del trabajo en la sala de clases. En este contexto, las historias familiares de los niños son asumidas como propias.

"Creo que aqui lo que caracteriza es que es como una familia para los niños, entonces eso hace que los niños vengan contentos al colegio que se atiendan sus necesidades tanto pedagógicas, también dentro de los que nosotros podemos, emocionales, de carencias que ellos tienen porque la mayoría de los niños vienen de familias que no son constituidas que tienen carencias emocionales importantes que obviamente afectan su desempeño pedagógico entonces yo creo que este es como el mejor sello de la escuela». (Profesora, Part-subv3, página 13).

Se destaca que los estudiantes de este establecimiento deseen asistir a la escuela. ¿En qué medida esta posición del profesor no sigue la lógica del cuasi-mercado y de las evaluaciones ministeriales?

Los entrevistados mencionaron mayoritariamente prácticas y valores que ellos esperan de sus alumnos: la felicidad y la práctica de valores positivos. La combinación de ambos sería el reflejo de una acción concreta concebida en la escuela. En esta escuela municipal, un docente nos dice:

«La prioridad es que el niño se sienta feliz, que esté progresando en elementos para desarrollar su personalidad, valores, que los tengan fuertes, que los practiquen, o sea que, si es honrado, que lo practique, que no lo diga solamente. Que devuelva un billete, un celular, aunque cueste \$300.000 pesos». (Profesor, Mun2, página 9).

La honradez es un aspecto en cuestión en ciertas creencias sobre la cultura de los barrios pobres. El estigma del narcotráfico y la delincuencia han generado una atención mayor en las escuelas que deja de lado a otros requerimientos.

Las carencias emocionales evocadas responderían a vacíos que se producen en sus ambientes, evidenciando ausencias familiares o episodios de violencia al interior del hogar: «... porque uno es poco lo que se puede enseñar, por la variedad de niños y las historias que tienen detrás, entonces un poco más de cariño más que tanto enseñarles...». (Profesora, Mun1, página 11).

«... es poco lo que se puede enseñar...» Esta sentencia relativiza, a nuestro pensar, la idea de performatividad (Ball, 2003; Luengo y Saura, 2013): ella se refiere a generar un rendimiento sostenido en el tiempo con fuerte orientación a la mejora de resultados. El cariño aparece como un elemento foráneo a la performatividad (Ibid), puesto que no responde una proyección de futuro o de logro, sino que su práctica no posee una temporalidad evolutiva, ni se mide bajo un estándar.

\subsection{Convivencia escolar}

La violencia referida en las entrevistas da cuenta de un panorama complejo. En muchos de estos sectores, el narcotráfico se ha tomado los barrios minando en gran parte el tejido social poblacional. 
Sumado a esto, las pocas instituciones públicas y servicios privados que se instalan en estos sectores, revelando un abandono tanto del Estado como del mercado. Situación que se ve más claramente en el Castillo (Ortega, 2014).

«Son todos de la población, no hay que olvidar nunca eso, que pese a todas sus deprivaciones, llegan a acá». (Profesora, Mun1, página 19).

«Aqui en las poblaciones acá cercanas, mucho tráfico, balaceras, o sea es una realidad complicadita... a mí el año pasado me tocó que un niño viniera con pistola, con cuchillo, y tú tienes que ponerte serio». (Profesora, Mun1, página 19).

«Donde yo vivo, por aquí también, viste, las calles no tienen ni nombre porque asi no pillan a nadie». (Profesora, Mun2, página 20).

El trabajo de reducción de los focos de conflicto presente en las escuelas hace aparecer iniciativas propias que los equipos de trabajo discuten e implementan. Mencionaremos dos planes llevados a cabo:

- Planes de normalización: plan que se centra en respetar las fechas y los horarios de las actividades planificadas.

- Recreos dirigidos: llamado en una de las escuelas visitadas recreos entretenidos. Este plan, más específico se concentra en las pausas durante la jornada escolar.

La búsqueda de la cultura de la paz se posiciona como un ideal preponderante mucho antes que cualquier resultado escolar medible y comparable. En este punto, apreciamos coincidencias entre las escuelas particulares subvencionadas y municipales.

\subsection{EL SIMCE y los resultados escolares}

Los entrevistados nos expresaron principalmente una relación ambivalente con esta prueba. En un primer momento, se le cataloga como homogeneizador que no logra atender a todas las particularidades de los niños, ya que su objetivo es medir los aprendizajes de ciertos niveles de la escolaridad, entregando puntajes promedios por establecimiento. En tal sentido, los profesores develaron un contexto en el que se debe atender a alumnos muy distintos entre sí, mostrando una diversidad que va más allá de las clasificaciones de alumno prioritario, o tan solamente, pobre. Un docente de escuela municipal nos dice: «El SIMCE está hecho una prueba para todos igual, y a los niños de acá siempre les va a ir mal porque no saben responder a eso». (Profesora, Mun1, página 8).

$\mathrm{Al}$ mencionarse una prueba para todos por igual se demuestra que en esta política pública de alcance nacional, que ordena el cuasi-mercado mediante una clasificación de las escuelas con un criterio que despersonaliza al alumno y hace emerger a la escuela como primer ente evaluado. Aquí, la lógica del estándar es percibida claramente por este docente.

Ante esto, los docentes tomas diversas posiciones: «acá, el SIMCE no es tema, de hecho, por eso nos criticaron del ministerio: ustedes son un colegio, no un hogar... nos guste o no, nos miden igual, como una escuela más». (Profesora, Part-subv3, página 7).

Aquí, aparece una resignación ante el SIMCE. La autonomía de la escuela, uno de los pilares de la política escolar chilena, no tiene primacía cuando un establecimiento persigue otros fines. Por tanto, 
dentro de una lógica de Estado evaluador (Maroy, 2009; Falabella, 2015), la autonomía de las escuelas tiene límites que van más allá de lo previsto incluso por la misma constitución política de la nación ${ }^{16}$.

Sin embargo, en una de las escuelas particulares subvencionadas, el SIMCE está absolutamente integrado al plan de estudios de la institución. Este hecho da cuenta que pese a no existir una valoración, al menos declarativa, sobre la prueba nacional, este instrumento forma parte de la cultura escolar y es difícil concebir la acción educativa sin mirar al SIMCE como referente. Un profesor de un establecimiento particular subvencionado nos indica: «se arrojan las hojas de respuesta a la máquina y te arroja las respuestas que más malas tuvieron, las mejores, entonces con respecto a eso uno va tomando decisiones». (Profesora, Part-subv2, página 8).

En estos sectores, los resultados del SIMCE son históricamente bajos y muestran que no existe una presión fuerte de parte del poder central, esto se ve refrendado por un tipo de apoderado ${ }^{17}$ que no se mueve mayormente, matriculando a sus hijos en la misma escuela (Córdoba, 2014). Esto llevaría a las escuelas a centrarse en áreas distintas a las del rendimiento escolar, aunque no podemos concluir que esto último haya desaparecido de las agendas de las escuelas.

\subsection{Rendición de cuentas}

Este proceso reviste una singularidad notoria. A la luz de las entrevistas, la rendición de cuentas merece ser interrogada sobre su naturaleza (¿Qué cuentas rendir?); los actores involucrados (¿Quién(es) rinde(n) las cuentas? ¿Para quién(es)?); así como de las instancias (¿Dónde y cuándo rendir las cuentas?). En base a estas simples preguntas, intentaremos reconstruir el discurso del profesorado.

\subsection{La naturaleza de la responsabilidad en las escuelas}

Dos términos íntimamente ligados pueden permitirnos un acercamiento a la rendición de cuentas. En primer lugar, el desempeño escolar (Bellei y González, 2003), el cual hace referencia a la relación proceso-resultado fijado por la política educativa. Por otro lado, la responsabilidad individual (Luengo, y Saura, 2013). Ésta se refiere a las tareas que son asumidas por cada ente de la comunidad escolar y abarca tanto la ejecución de la tarea como las consecuencias de su aplicación.

En las escuelas visitadas asomó la segunda terminología como una forma de representar la rendición de cuentas: la repartición de responsabilidades de los equipos educativos y de los apoderados se asomó como un principio clave.

Un docente de una escuela particular subvencionada nos dijo: «... en el colegio, es una responsabilidad compartida entre apoderados y colegio y ellos es prácticamente "abi está mi hijo, yo se los entrego a ustedes, háganse cargo" la mayoría es asír. (Profesora, Part-subv1, página 21).

El primer punto sobre la responsabilidad es definirla como compartida, sin embargo, se señaló que las familias se sienten en una relativa comodidad, puesto que la escuela se ocupa de atender a sus hijos. Este comportamiento puede exhibir un rol de consumidor de la educación y el papel que desempeñan las familias de barrios pobres, en el cuasi-mercado escolar chileno (Córdoba, 2014).

\footnotetext{
16 Sobre la libertad de enseñanza, la Constitución Política dice: La libertad de enseñanza no tiene otras limitaciones que las impuestas por la moral, las buenas costumbres, el orden público y la seguridad nacional. Capítulo III: de los derechos y deberes constitucionales, artículo 19, párrafo 11.

17 Apoderado: denominación usada en Chile para referirse a los padres, familiares o tutores que representan y/o acompañan al alumno en su escolarización. Él asiste a las reuniones mensuales, llamadas de apoderados.
} 
De todos modos, en otra escuela particular subvencionada nos dice más al respecto: «no podemos ponerles un dos por no traer materiales, si no es culpa del niño, es culpa de los adultos, entonces, hay varias entrevistas con los papás». (Profesor, Mun2, página 22).

La falta de material escolar pedido para ciertas actividades se adjudica a una despreocupación de los padres más que al olvido del niño. Esto motiva a reunirse con estos apoderados a fin de solucionar este tipo de situaciones.

En las escuelas municipales, se percibe una situación parecida. Una profesora nos señala: «... nosotros podemos hacer mucho, pero si la familia no hace nada...». (Profesor, Mun2, página 23).

El entrevistado nos expresa que se espera, de parte de la escuela, una mayor atención y colaboración de las familias. Sin embargo, las vivencias relatadas por los docentes sobre la cultura y el contexto de las poblaciones parecerían generar una gran contradicción acerca de si efectivamente el apoderado está en condiciones de asistir a la escuela en la labor formadora que ésta dice ejecutar. Como lo refrenda otro docente: «bay algunas mamás que no saben escribir y no saben leer, entonces no les puedes poner un PowerPoint o cuando pasamos las nóminas hay que decirles donde firman». (Profesora, Mun1, página 23).

El analfabetismo funcional fue identificado en algunos casos como factor que incide fuertemente en la comunicación que se efectúa entre familia y escuela.

Por ende, construir una convivencia tiene que ver con generar una mayor participación: «considero que la prioridad es mantener una sana convivencia y en la medida en que haya una sana convivencia, se logran buenos aprendizajes». (Profesora, Part-subv4, página 9). Este posicionamiento muestra como la prioridad de esta escuela pasa por mantener un buen clima de las relaciones y que por añadidura vendrán los buenos resultados. Solo una escuela, particular subvencionada, se enfocó casi únicamente en los contenidos:

«Son hartas tareas que de repente uno como profesor jefe tiene que hacer, revisar que la agenda esté firmada, revisar que, porque aqui se hacen guías a los 15 minutos, de lenguaje y de matemáticas, entonces es mucho lo que uno tiene que abarcary poco tiempo... eso implica otra hora más en donde tienes que detenerte aún y eso es lo que aquí no se puede hacer». (Profesora, Part-subv1, página 10).

Detenerse en los contenidos no parece ser una tarea prevista: aquí, la performatividad ocupa un rol no menor en cuanto a su ritmo hacia adelante. La relación tiempo-actividad en esta escuela apuesta por una carga de trabajo importante.

\subsection{La responsabilidad de los actores involucrados}

A nivel de las escuelas particulares subvencionadas se comparte la idea de articulaciones entre actores. La comunicación entre pares se define dentro de un plan preestablecido de responsabilidad escolar:

"Un equipo que se preocupa, que está constantemente preguntando cómo van, qué dificultades están teniendo, de hecho, aqui la modalidad es que ellos trabajen por ejemplo si yo soy la profesora de matemáticas y le hago al sexto c por ejemplo yo voy y tengo contacto directo con el profesor jefe de ese curso y le estoy constantemente diciendo sabes que este niño lleva dos semanas sin responder con las tareas». (Profesora, Part-subv1, página 16).

La gran idea fuerza expresada por los docentes de escuelas municipales es disponer de un sistema altamente organizado, que esté acorde a los requerimientos de los niños de la población. 
La privatización de la educación en Chile: análisis de los discursos del profesorado y de la dirección de los centros sobre la calidad de la educación y la rendición de cuentas

La presencia del director en los espacios del establecimiento escolar es destacada por los entrevistados: «este director es de patio. El estar alli con los niños...que esté con los niños, que se involucre que los niños sepan que está abi para ayudarlos». (Profesora, Mun1, página 16).

De este modo, el tiempo destinado a estar en los lugares donde los niños se concentran, por ejemplo, el recreo, genera una buena apreciación por parte de los entrevistados y determina, por ende, la responsabilidad. La presencia real es altamente valorada.

\subsection{Instancias de rendición de cuentas}

La reunión de apoderados emerge como un instante de comunicación donde tanto profesores como apoderados se informan de la situación de los niños. Estas reuniones pueden ser con el total de apoderados de un curso o con uno en particular. Así lo refrenda un entrevistado: «bay apoderados y apoderados, unos que se molestan de que los sigan llamando siempre, pero si yo los cito es para ver algún avance, para que ellos se hagan cargo del problema». (Profesora, Part-subv4, página 16).

La citación de apoderados es, en particular, en este colegio un recurso muy utilizado. Ya sea para mostrar los avances o para identificar un problema. En ambos caso subyace la mejora o progreso, además de constatarse un estado presente deficitario. Se refleja un desarrollismo hacia el futuro en el tratamiento del comportamiento.

La reunión de apoderados constituye el espacio de carácter asambleísta tradicional de las escuelas chilenas y se presenta como el momento donde el profesor jefe explica la situación del curso y trata temas tanto organizativos como conductuales y académicos, sin embargo el contexto también incide, en palabras de un docente: «en las reuniones, por ejemplo, tengo a la mitad de apoderados y a la mitad niños porque no los quieren dejar en sus casas solos porque de repente puede pasar algo». (Profesora, Part-subv3, página 22).

Las causas de no asistir a la reunión de apoderados son urgentes y no se relacionan necesariamente con el avance o retroceso de los comportamientos de los niños en las escuelas.

Del mismo modo, las reuniones de trabajo entre equipo directivo y docentes es otro espacio donde se comunican situaciones del diario vivir de la escuela, así como se proponen nuevas ideas. Según una profesora: «el dire (Director) ha hecho hartas cosas... tenemos un director que nos anda...no... en las reuniones nos dice, chiquillos, tenemos que hacer esto, esto otro». (Profesora, Mun2, página 16).

La rendición de cuentas se observa como reiterados momentos y espacios de comunicación entre los miembros de la comunidad escolar. Como lo mencionamos, el avance o mejora de los comportamientos revela una idea de rendimiento performativo con proyección en un futuro. Sin embargo, las conversaciones entre miembros del establecimiento y las condiciones sociales de la población ofrecen una interpretación ajena a la mera mecánica de un proceso performativo que busca resultados.

\section{Modos de gestión en contextos de pobreza y reconfiguración de la profesión docente}

\subsection{Modos de gestión en contextos de pobreza}

En esta parte nos centraremos en el discurso de los directores de escuela. Hemos seleccionado algunos párrafos del capítulo octavo de nuestra investigación antes nombrada y que se enmarca en el trabajo de tesis doctoral de uno de los autores del presente artículo. Aquí, asomó un consenso general sobre la constitución de equipos de gestión. Estos grupos se estructuran según organigramas dispuestos y separados por ciclos de aprendizaje de la enseñanza básica, encabezados por el equipo de gestión. 
Comenzando por las escuelas particulares subvencionadas, un director establece un punto de partida: «(la gestión es) Horizontal, el organigrama está dividido por ciclos... ¿Qué tiene que hacer el jefe de nivel, jefe técnico, la subdirectora? Velar que los acuerdos se cumplan». (Director, Part-subv1, página 11).

Aquí vemos cómo la delegación de las decisiones implica a puestos de administración (subdirección), así como pedagógicos (jefe de nivel y jefe técnico). Se produce una subsidiaridad de las decisiones al interior de la organización (Igalens et al., 2002).

En las escuelas municipales, la confección de equipos de tres personas: director, jefe de UTP e inspector general, confirma una forma de organización común, que no tiene mayores diferencias; sin embargo, los requerimientos del contexto mostraron que el organigrama clásico no siempre responde por sí solo a una institución:

«La toma de decisiones abarca más personas, primero un equipo: aqui partimos con tres personas, a pesar de que no existía inspector general, imaginate una escuela dramática como ésta y no teníamos inspector general, por 4 años ó 5, entonces las labores las haciamos tres personas: el director, la jefa de UTP y la orientadora». (Director, Mun2, página 6).

La situación dramática enunciada se refiere al conflicto social que se vive en el entorno, donde la percepción de violencia en las calles y presencia de narcotráfico se encuentra presente de manera directa (Cornejo, 2012).

En una escuela particular subvencionada se nos dijo:

«Nos hace también pensar en algún momento que los delincuentes estaban en el interior de las mismas familias de nuestros niños, entonces cómo pasar el mensaje a ellos, que no nos bicieran el daño, y eso nos ha llevado a tener mucha cercanía con la gente del sector». (Directora, Part-subv3, página 6).

Por tanto, la organización de las escuelas es tradicional, lo que se modifica es la prioridad que se atiende.

En efecto, ¿Qué puede hacer la escuela? Un director de escuela municipal nos detalla cómo procedieron:

«Se veía el estado académico, como también todas sus problemáticas de necesidades educativas, como también psicológicas, y de salud [...]mejorar el trato de atención tanto a los alumnos, como a los apoderados, expedito, aplicar los manuales de convivencia como corresponde, darle buena respuesta en los conflictos, mediar en los conflictos». (Director, Mun2, página 7).

Por último, las redes y la gestión de proyectos fue un elemento significativo de las entrevistas. Lo que puede parecer un beneficio para la comunidad escolar en contextos vulnerables: la conexión con actores locales en contextos donde la economía informal tiene gran presencia, dado la falta de institucionalidad pública y del mercado:

"Uno puede decir, pero Ud. ¿Por qué no combate a la señora que vende los calzones rotos afuera o la sopaipilla, pero iesabe qué?! Si yo hago eso me van a surgir cuatro negocios más. Y ellas son colaboradoras, son nuestro mejor filtro, porque conocen a toda la gente de acá y eso me dice: "abi hay cuatro jóvenes que no son de acá, deben estar esperando a alguien». (Director, Mun1, página 12). 
Estas colaboradoras, que se ganan la vida en la venta de comida casera, representan una ayuda. La percepción de los directores, en especial en el Castillo, es de un abandono del Estado que no piensa directamente en la escuela de zonas pobres de la periferia. Este mismo director de escuela municipal da un veredicto muy certero:

\section{"Cuando tomen decisiones de la política educacional de Chile, salgan del anillo de Américo Vespucio, porque está pasando algo distinto que las autoridades no están viendo, si yo pienso políticas de Estado pensando en el Instituto Nacional, esto no va a cambiar». (Director, Mun1, página 3).}

Los modos de gestión en las escuelas visitadas dan cuenta de un organigrama clásico de las escuelas chilenas (equipos de gestión, jefes de nivel, etc.), sin embargo, las prioridades se enfocan en gran medida en las necesidades o carencias de los estudiantes y familias que se escolarizan. La consecución de resultados medibles y estandarizados, a nivel nacional, se reordena, en función de este objetivo primordial. Las necesidades del contexto, que acarrean una historia propia, a través del tiempo -y también, fuera del tiempo- puesto que los barrios vulnerables o poblaciones poseen dinámicas internas que recrean realidades coetáneas a la ciudad misma; y en consecuencia, realidades coetáneas a nivel escolar, que repercute finalmente, en prioridades distintas a las dispuestas por la política educativa nacional.

\subsection{Reconfiguración de la profesión docente}

La profesión docente, la abarcaremos mayormente desde el papel que los profesores juegan en los establecimientos visitados, descrito por los directores. En un primer lugar, asoma como elemento común en las entrevistas, el rol del docente en la escuela y su implicación en las prioridades del establecimiento. En una escuela particular subvencionada, la directora nos dice:

«No tenemos bullying, no tenemos niños agresivos, niños atrevidos, no hay problemas de disciplina, se trabaja súper bien con un equipo que está contento de venir a trabajar... es difícil encontrar trabajadores que estén felices de venir a su trabajo, a niños felices de venir a la escuela, eso lo tenemos logrado, el ministerio comenzó a valorar eso». (Directora, Part-subv3, página 4).

La violencia escolar afecta a toda y todos los miembros de la comunidad escolar y se materializa de distintas maneras. Para hacerle frente, la estrategia desplegada en la escuela es la práctica de buenas relaciones entre personas para, de este modo, disminuir los focos de conflicto que puedan existir.

Este plan se ve confirmado en otro establecimiento particular subvencionado, su director nos declaró:

"Aquí, la formación de profesores se demoró mucho tiempo. El profesor está formado aquí, llegaron siendo ayudante de curso, siendo estudiantes, eran estudiantes de la universidady llegaron aqui... entonces ha habido una forma de aprendizaje dentro de la escuela, que es el respetar a los niños, para que los niños nos respeten, entonces se da ese aprender... eso significa trabajo, abi hay una relación buenay hay que desarrollar acción, tengo que mostrarles una actitud». (Director, Part-subv1, página 10).

El director habla de una formación por el trabajo del docente. Lo cual genera un sello propio de este establecimiento que comienza por el respeto hacia los niños. Desde esta mirada, se estructuran los aprendizajes y las metodologías.

Un director de escuela municipal nos señala este paso de cultura de violencia a espacio de atenuación: 
«Pasar de una sala de clase en la cual se burlaban de eso, poniéndoles cosas en la silla, escupos, sacándoles la cartera, escondiéndoles el libro de clase, insultándoles, escapando por la ventana, a una sala de clase contenedora, donde uno podía ejercer su profesión, para lo que está mandatada, pasar a un curso donde ahora se puede hacer clase». (Director, Mun2, página 5).

La organización de los horarios se presenta como la primera determinación: el plan basado en hacer las actividades planificadas, según los horarios previstos, da cuenta de una disciplina escolar inicial, sin embargo, el planteamiento de estas medidas es bajo el enfoque del buen clima y los entendimientos.

Siguiendo esta línea de la sala de clases como lugar central del ejercicio docente, ante esto, los episodios de conflicto entre alumnos, son enfrentados en busca de minimizarlos, el objetivo es optimizar los tiempos de aula, con miras a normalizar el quehacer escolar: el director de una escuela particular subvencionada nos dice: «... hemos fortalecido las clases: que el profesor siempre esté en el aula». (Directora, Part-subv4, página 18).

El tiempo que el docente pase en la sala de clase define mucho para los directores. Esto se transforma en un indicador de avance de los proyectos emprendidos tendientes a superar los conflictos. Ahora bien, el docente puede vivir dificultades. Ante esto, los equipos educativos cobran relevancia, un director de escuela municipal lo justifica: «fuimos incorporando más gente... porque los profesores fundamentalmente en la escuela pública, están solos y lo niños que son más disruptivos, no tienen solución...». (Director, Mun2, página 16).

Por lo tanto, asistimos a procesos, tanto de inserción docente como consolidación profesional, desde un enfoque de la resolución de conflictos. Ahora bien, el foco sobre el acompañamiento y la conversación continua puede tender, no necesariamente a disciplinar a los alumnos, entendiendo este término como el ordenamiento tanto físico como mental de los niños según los requerimientos de la cultura escolar, sino que puede abrir espacios a finalidades distintas a la sola evaluación de resultados académicos: una institución que busque mejores relaciones humanas puede constituir este propósito como su propio sello de calidad.

Apreciamos también una desconexión con la vida en las poblaciones. Así nos lo dijo una directora de escuela particular subvencionada:

«Si nos midieran en otros aspectos, nos iría regio, pero a nadie le importa lo que hacemos... creo que falta bumanidad, fobia escolar, estrés infantil, excesivas guías de trabajo para sábado o domingo, llegan odiando la escuela, llegan enfermos. Entonces, nuestro rol es sanarlos, y hay resultados: vienen niños que están expulsados, con problemas conductuales, que aqui se portan bien... se sienten queridos, aceptados y nadie los trata malis. (Directora, Part-subv3, página 16).

La precariedad social se asocia a una responsabilidad del Estado, dejando la excelencia y la preocupación por los resultados a las escuelas privadas, pero nuestro ejemplo, muestra lo contrario: un privado que se ocupa de esto último, dejando en segundo plano los resultados. Sin embargo, esto no está en sintonía con el enfoque de la eficiencia de los resultados.

\section{Conclusión}

En presente artículo hemos intentado reflexionar sobre el proceso de privatización de la educación en Chile, desde la perspectiva de la idea de la calidad de la educación y de la rendición de cuentas, en sectores pobres del país. 
La privatización de la educación en Chile: análisis de los discursos del profesorado y de la dirección de los centros sobre la calidad de la educación y la rendición de cuentas

En los sectores populares se aprecia una alta privatización exógena o privatización de la educación, llegando, en algunos casos, La Pintana, al $80 \%$ de la matrícula escolar concentrada en escuelas particulares subvencionadas. Constituyendo un caso significativo de presencia de provisión privada en una de los sectores más pobres del país.

Sin embargo, al hablar de las formas endógenas de privatización o privatización en la educación, el análisis resulta menos nítido que en el primer caso: las entrevistas realizadas en establecimientos particulares subvencionados y municipales nos permiten señalar la presencia de combinaciones complejas de valores y prácticas venidas de la cultura privada; así como de ideas asociadas tradicionalmente a lo público. Generando, de este modo, verdaderas hibridaciones o tránsitos de fronteras público-privado.

La convivencia escolar busca atender las dificultades sociales de los niños y generar una incorporación mayor de los apoderados. Este trabajo tomaría, sin embargo, algunas lógicas más asociadas a la privatización endógena: tal es el caso del avance o estancamiento del rendimiento conductual, lo que supone una mirada de evolución hacia el futuro de los comportamientos, idea que asocia a la performatividad de los sistemas escolares. Sin embargo, el cariño y la proximidad con los niños relativizan la idea anterior.

El SIMCE, por su parte, ocupa un lugar secundario, casi externo a los objetivos propios de los establecimientos. Los requerimientos de la población copan gran parte de las tareas desempeñadas por los docentes. Así, la calidad basada en resultados no se refiere esencialmente a la obtención de buenos escores SIMCE, sino más bien, a otros indicadores complementarios para la política educativa chilena, a saber, bajar las tasas de repetición y absentismo escolar, prevenir conflictos y peleas, y aumentar la participación de las familias en las escuelas.

La rendición de cuentas se enfoca mayormente en la responsabilidad que cada actor juega dentro de la institución más que a exponer públicamente los resultados de una tarea empeñada. Las conversaciones diarias, los planes de convivencia propios, son instancias de repartición de roles.

La gestión posee un organigrama establecido y clásico a toda escuela, donde sus tres autoridades comparten un liderazgo que se enmarca más en la presencia dentro del establecimiento que en la exigencia jerárquica de tareas a los subalternos. La búsqueda de un clima escolar de paz ocupa nuevamente la prioridad.

El profesorado de las escuelas en sectores vulnerables transita de distintas maneras en las prácticas docentes frente a las políticas de rendición de cuentas según resultados. En las escuelas particulares subvencionadas, en general, apreciamos a docentes más centrados en los scores de los alumnos, aunque más bien se trata de resultados internos más que de la prueba SIMCE. En una caso, al tratarse de una escuela católica este objetivo se vincula mayormente con el ideario religioso de buen alumno, pero que en la práctica es concordante con la performatividad proclamada por el Estado. El profesorado de escuelas municipales -y de una escuela particular subvencionada- responde a una adaptación de los resultados, toda vez que estos incluyen la cercanía del docente con el niño en el diario vivir, generando planes de normalización y de orden buscan entregar mejores condiciones a los profesores y quitar del imaginario la reputación de las poblaciones aledañas a las escuelas.

Todo esto nos lleva a pensar en una discordancia entre las políticas educativas chilenas y las prioridades adoptadas por las escuelas de poblaciones de la Región Metropolitana, provocando espacios o vacíos, en una relación de centro - periferia o totalidad- exterioridad, tanto en el plano operativo como concep- 
tual en la asimilación de la calidad de la educación y en la rendición de cuentas. Estos vacíos generan una inmovilidad de los actores en el proceso escolar, más que nuevas perspectivas tanto pedagógicas como de la generación de proyectos educativos emancipatorios en estos sectores, de los más pobres de la Región.

\section{Referencias bibliográficas}

Aedo, Cristián y Sapelli, Claudio (2001). El sistema de vouchers en la educación: una revisión de la teoría y la evidencia empirica para Chile (N. ${ }^{\circ}$ 307). Centro de Estudios Públicos.

Akkari, Abdeljalil; Pompeu, Camila; Fernandes Costa, Ana Sheila y Mesquida, Peri (2011): "Construction historique d'un système éducatif dual et brouillage actuel des frontières entre les réseaux scolaires public et privé au Brésil". Revue Autrepart, 59, 109-124.

Almonacid, Claudio. (2004): "Un cuasi mercado educacional: la escuela privada subvencionada en Chile". Revista de Educación, 333, 165-196.

Almonacid, Claudio (2008): "La educación particular subvencionada como cooperadora del Estado" en Bellei, Cristián, Contreras, Dante y Valenzuela, Juan Pablo (Comps.): La Agenda Pendiente en Educación. Profesores, Administradores y Recursos: Propuestas para la Nueva Arquitectura de la Educación Chilena. Santiago: Programa de Investigación en Educación Universidad de Chile/UNICEF.

Ball, Stephen (1990). La micropolitica en la escuela. Barcelona: Paidós

Ball, Stephen, (2003): "Profesionalismo, gerencialismo y performatividad". Revista Educación y pedagogía, 15 (36), 85-104.

Ball, Stephen y Youdell, Deborah (2008). La privatisation déguisée dans le secteur éducatif public. Bruxelles: Internationale de l'éducation.

Bellei, Cristián y González, Pablo (2002). "Educación y competitividad en Chile”, Documento de trabajo, Santiago de Chile, Facultad Latinoamericana de Ciencias Sociales (FLACSO).

Bellei, Cristián, et al., (2003): "Escuelas efectivas en sectores de pobreza". La educación en Chile, hoy, 347372.

Bellei, Cristián., et al., (2015). "Nadie dijo que era fácil." Escuelas efectivas en sectores de pobreza, diez años después [No One Said it is Easy. Effective School in High Poverty Areas, Ten Years Later]. Santiago, (Chile): LOM

Bellei, Cristián (2016): “Dificultades y resistencias de una reforma para des-mercantilizar la educación”. RASE: Revista de la Asociación de Sociología de la Educación, 9 (2), 232-247.

Carnoy, M. (1998): "National Voucher Plans in Chile and Sweden: Did privatization reforms make for better education?" Comparative education review, 42 (3), 309-337.

Constitución Política de la República de Chile. Diario Oficial, Santiago, Chile, 24 de Octubre de 1980.

Córdoba, Claudia (2014): "La elección de escuela en sectores pobres: Resultados de un estudio cualitativo”. Psicoperspectivas, 13 (1), 56-67.

Cornejo, Catalina Andrea (2012): "Estigma territorial como forma de violencia barrial: El caso del sector El Castillo”. Revista Invi, 27 (76), 177-200. 
Cox, Cristián (2003): "Las políticas educacionales de Chile en las últimas dos décadas del siglo XX". Politicas educacionales en el cambio de siglo. La reforma del sistema escolar en Chile, 19-113.

De Sousa Santos, Boaventura (2010). Descolonizar el saber, reinventar el poder. Montevideo: Ediciones Trilce.

Decreto Ley N. ${ }^{\circ}$ 3.477. Diario Oficial, Santiago, Chile, 2 de Septiembre de 1980.

Elacqua, Gregory y Santos, Humberto (2013): "Preferencias reveladas de los proveedores de educación privada en Chile: El caso de la Ley de Subvención Escolar Preferencial”. Gestión y política pública, 22 (1), 85-129.

Falabella, Alejandra (2015): "El mercado escolar en Chile y el surgimiento de la Nueva Gestión Pública: El tejido de la política entre la dictadura neoliberal y los gobiernos de la centroizquierda (1979 a 2009)". Educação \& Sociedade, 36 (132), 699-722.

Garretón, Manuel Antonio; Cruz, María Angélica; Aguirre, Félix; Bro, Naim; Farías, Elías; Ferreti, Pierina y Ramos, Tamara (2011): "Movimiento social, nuevas formas de hacer política y enclaves autoritarios: Los debates del Consejo Asesor para la Educación en el gobierno de Michelle Bachelet en Chile”. Polis (Santiago), 10 (30), 117-140.

Igalens, Jacques, Joras, Michel y Mancy, François (2002). La responsabilité sociale de l'entreprise: comprendre, rédiger le rapport annuel. Paris: Editions d'Organisation.

Kutscher, Macarena (2014). Financiamiento Compartido y su efecto en diferentes indicadores de calidad educativa. Documento de trabajo n. ${ }^{\circ}$ 1. CLAPES.

Larrañaga, Osvaldo (2004): “Competencia y participación privada: la experiencia chilena en educación”. Estudios Públicos, 96, 107-144.

Luengo, Julián. y Saura, Geo Saura. (2013): “La performatividad en la educación. La construcción del nuevo docente y el nuevo gestor performativo". Revista Iberoamericana sobre Calidad, Eficacia y Cambio en Educación, 11 (3), 139-153.

Luengo, Julián, et al., (2012): "Nuevas formas, nuevos actores y nuevas dinámicas de la privatización en educación”. Profesorado,16 (3), 3-10.

Ministerio de Educación. (2015). Estadísticas de Educación 2015. Recuperado de: https://centroestudios.mineduc.cl

Ministerio de Educación. (2008). Marco para la Buena Enseñanza. Recuperado de: https://www.google. $\mathrm{ch} /$ url? $\mathrm{sa}=\mathrm{t} \& \mathrm{rct}=\mathrm{j} \& \mathrm{q}=\& \mathrm{esrc}=\mathrm{s} \&$ source $=$ web\&cd $=1 \& v e d=0$ ahUKEwjpnLurgcrUAhUPfFAKHWs NCogQFggnMAA\&url=https\%3A\%2F\%2Fwww.docentemas.cl\%2Fdocs\%2FMBE2008.pdf\&usg= AFQjCNFxTAi3vDPY2yGZzdp91ct9CS224A\&sig2=MryUJEvFEyuPDbfG01Ak8g\&cad=rja

Moulian, Tomás (1994): “Limitaciones de la transición a la democracia en Chile”. Proposiciones, 25, 34-45.

Ortega, Tamara (2014): “Criminalización y concentración de la pobreza urbana en barrios segregados: Síntomas de guetización en La Pintana, Santiago de Chile”. EURE (Santiago), 40 (120), 241-263.

Torres, Carlos A. (2000). The State, Privatization and Educational Policy: A Critique of Neoliberalism in Latin America and Some Ethical and Political Implications. Los Angeles: University of California. 


\section{Notas biográficas}

Ignacio Eduardo Reyes Cayul es estudiante de doctorado en ciencias de la educación de la Universidad de Ginebra, su tesis trata sobre la escuela privada en contextos de pobreza en Santiago de Chile.

Con anterioridad, realizó estudios de licenciatura en educación y pedagogía en francés en la Universidad Metropolitana de Ciencias de la Educación de Chile, además de la maestría en ciencias de la educación: análisis de los sistemas educativos, en la Universidad de Ginebra.

Abdeljalil Akkari es Doctor en ciencias de la educación de la Universidad de Ginebra, actualmente se desempeña como profesor ordinario en dimensiones internacionales de la educación de la Facultad de Psicología y Ciencias de la Educación de la Universidad de Ginebra.

Responsable del Equipo de Investigación Dimensiones Internacionales de la Educación (ERDIE) su sigla en francés. Sus principales líneas de investigación son las dimensiones interculturales e internacionales de la educación, internacionalización de las políticas educativas, métodos comparativos en educación e investigación sobre la educación de base en los países del sur, entre otros temas. 